\title{
Nanotechnology in Dentistry - What does the Future Hold in Store?
}

Sivaramakrishnan SM and Neelakantan $\mathbf{P}^{*}$

Saveetha Dental College and Hospitals, Saveetha University, Chennai, India

\begin{abstract}
The medical and dental field has seen several technological revolutions that have changed clinical practice. One concept which has and holds further promise in bringing about a paradigm shift in the field of diagnostics and management is nanotechnology. Nanotechnology has several applications in dentistry, from diagnosis of pathological conditions to local anaesthesia, orthodontic tooth movement and periodontics. Biomaterials science has also greatly benefited by this technology. This review provides a comprehensive discussion of the present and future of nanotechnology in dentistry.
\end{abstract}

Keywords: Nanoparticle; Biomaterials; Nanomedicine; Nanodentistry; Nanorobots; Nanofillers

\section{Introduction}

The term nanotechnology was first introduced by Richard Feynman in 1959 to the scientific approach of creating functional materials, devices and systems through control of atoms on a nanometer scale and exploitation of novel phenomena and properties at that length scale [1,2]. This was practically made possible by Eric Drexler in the mid-1980s when he emphasized the potential of molecular nanotechnology [3,4]. The term "Nano" is derived from the Greek word "dwarf". More simply speaking, one nanometer is one-billionth or $10^{-9}$ of a meter [2,5-8]. What makes the concept of nanotechnology exciting is that their size is smaller than the critical lengths defining many physical events. With the application of nanotechnology in the medical field, it would be possible to customize the diagnostics and treatment plans based on the genetics of each patient $[9,10]$. Nanotechnology is very diverse, ranging from extensions of conventional device physics to completely new approaches based upon molecular self-assembly dealing with development of new materials to investigating whether we can directly control matter on atomic scale [11]. The technology can be comfortably applied to the fields of medicine and dentistry to yield the terms nanomedicine and nanodentistry respectively. The objective of this short review is to discuss what nanotechnology holds for dental practice in future.

\section{Approaches in Nanotechnology}

Three approaches have been followed in production of nanoparticles, namely Bottom up approach, Top down approach and functional approach [12]. The functional approach disregards the method of production of a nanoparticle, and the objective is to produce a nanoparticle with a specific functionality.

The fields of science and technology have witnessed the fabrication of several nanoparticles that we come across and use in our day to day lives, many a times not realizing it is part of the future revolution. The various nanoparticles are nano pores, nanotubes, quantum dots, nanoshells, dendrimers, liposomes, nanorods, fullerenes, nanospheres, nanowires, nanobelts, nanorings, nanocapsules [13]. This list of nanoparticles is by no means exhaustive.

\section{Nanotechnology in Dentistry}

Nanodentistry will make the maintenance of near-perfect oral health possible by the use of nanomaterials, biotechnology and nanorobotics [14].

\section{Nanodiagnostics}

This technology will help in the use of nanodevices for early disease identification at cellular and molecular level. The efficiency and reliability of diagnostic methods using human fluids or tissue samples by using nanodevices could be increased by nanomedicine [14]. In the case of in-vivo diagnostics, it could develop devices which will be able to work inside the human body in order to identify the early presence of disease, tumor cells and also identify and quantify toxic molecules [15]. Nanoelectromechanical systems and cantilever array sensors could be the methods of the future in the detection of cancer, bacteria, fungi and viruses [16]

A multidisciplinary team in Massachusetts institute of technology has developed carbon nanotubes that can be used as sensors for cancer drugs and other DNA damaging agents inside living cells. Dendrimers and nanoshells will facilitate drug delivery for targeted delivery to cancer cells.

\section{Nanotechnology in oral and maxillofacial surgery}

Local anaesthesia: Most dental procedures involve administration of local anaesthesia and several patients defer their dental treatment for the fear of injections. Truly painless methods of administration of local anaesthesia may be achievable by the application of nanotechnology. Hypothetically, a colloidal suspension containing millions of active analgesic micron-size dental robots will be instilled on the patient's gingiva. After contacting the surface of the crown or mucosa, the ambulating nanorobots would reach the pulp via the gingival sulcus, lamina propria and dentinal tubules guided by chemical gradients and temperature differentials under the control of the dentist with the help of nanocomputer $[14,16]$. Once installed in the pulp, these robots may shut down all sensitivity in any particular tooth that requires treatment. After the procedures are completed, these nanorobots may be manipulated to restore all sensation and relinquish control of nerve traffic and egress from the tooth by similar means used for ingress.

\section{Nanotechnology in orthodontic treatment}

Sliding a tooth along an arch wire involves frictional type of force

*Corresponding author: Dr. Prasanna Neelakantan, 1500, 16th Main Road, Anna Nagar West, Chennai - 600040, Tamil Nadu, India, Tel: +91 9884754914; E-mail: prasanna_neelakantan@yahoo.com

Received December 17, 2013; Accepted Janaury 23, 2014; Published January 25,2014

Citation: Sivaramakrishnan SM, Neelakantan P (2014) Nanotechnology in Dentistry - What does the Future Hold in Store? Dentistry 4: 198. doi:10.4172/2161 1122.1000198

Copyright: (C) 2014 Sivaramakrishnan SM, et al. This is an open-access article distributed under the terms of the Creative Commons Attribution License, which permits unrestricted use, distribution, and reproduction in any medium, provided the original author and source are credited. 
that resists this movement. Use of excessive force might cause root resorption and loss of anchorage. By coating the orthodontic wire with inactive fullerene-like tungsten disulfide nanoparticles known for their excellent dry lubrication properties, a reduction in this friction was observed by Redlich et al. [17]. In future, these nanorobots could directly manipulate periodontal tissues thus allowing a rapid, painless tooth straightening, rotating, vertical repositioning within minutes to hours.

\section{Nanotechnology in prosthodontics}

Impression materials: Nanofillers are integrated in vinyl poly siloxanes, thus producing unique addition silicone impression materials. This material is claimed to have better properties such as flow, adhesiveness. It also has improved hydrophilic properties hence fewer voids at margin and better model pouring and enhanced detail precision [9].

Implants: The most frequent cause of failure of implants is insufficient bone formation around the biomaterial immediately after implantation, with coating of nanoparticles over the dental implants, adhesion and integration to surrounding tissues is improved [1]. The surface of the implant which may be 'macro', 'micro' or 'Nano' structured, plays a main and critical role in determining biocompatibility and bio integration because it is in direct contact with the tissues. The nanostructured materials can exhibit enhanced mechanical, electrical, magnetic, optical properties compared with the conventional micronscale or macro -scale counterparts [18].

\section{Nanotechnology in periodontics}

Dentinal hypersensitivity: Natural hypersensitive teeth have eight times higher surface density of dentinal tubules and diameter twice as large as non-sensitive teeth. Dental nanorobots could selectively occlude these tubules within minutes and thus offers patients a quick and permanent cure [16]. These nanorobots reach the dentinal tubules and proceeds toward the pulp, guided by chemical gradients, temperature differentials, all under the control of nanocomputer. They can reach the pulp in approximately 100 seconds thereby offering a rapid relief of sensitivity [19-21].

Drug delivery: Periodontal therapy requires local delivery of drugs for more predictable results of treatment. Drug delivery systems based on triclosan incorporated nanoparticles have been developed. Pinon-segundo et al. have produced triclosan-loaded nanoparticles by the process of emulsification-diffusion, in an attempt to obtain a novel delivery system for the treatment of periodontal disease [22]. Tetracycline based microspheres are also being evaluated for placement in periodontal pockets.

\section{Nanotechnology in conservative dentistry and endodontics}

Tooth durability and appearance: Durability and appearance of tooth may be improved by replacing enamel layers with covalently bonded artificial materials like diamond and sapphire. They have 20 times more hardness and failure strength than the conventional ones and are also biocompatible. Nanorobotic dentifrice (dentifrobots) delivered by mouth wash or tooth paste could prevent accumulation of supra and subgingival calculus and may also metabolize the trapped organic matter into odorless, harmless vapors and aid in calculus debridement. They measure 1-10 micron and also may have the property to deactivate them if swallowed.

Nanocomposite resins: Non-agglomerated discrete nanoparticles that are homogeneously distributed in resins or coatings to produce nanocomposites have been successfully manufactured by Nanoproducts Corporation. The nanofiller used: Aluminosilicate powder with a mean particle size of 80 ran 1:4 $\mathrm{M}$ ratio of alumina to silica and a refractive index of 1.508. These nanocomposites have superior hardness, flexural strength, modulus of elasticity, decreased polymerization shrinkage and also have excellent handling properties [23]. A recent study by $\mathrm{Xu}$ et al. has evaluated the incorporation of nanosized $\mathrm{CaPO}_{4}$ particles into resin based-composites, with a resulting improvement in stress bearing capacity as well as ion release that could inhibit dental caries [24,25].

Nanoparticulate based disinfection in endodontics: The efficacy of nanoparticles to disinfect root canals has gained popularity in the recent past. This has been attributed to the broad spectrum of spectrum antibacterial activity [26,27]. The nanoparticles evaluated on endodontics include Chitosan, zinc oxide and silver [28,29]. The efficacy of chitosan and zinc oxide nanoparticles against Enterococcus fecalis has been attributed to their ability to disrupt the cell wall. In addition, these nanoparticles are also able to disintegrate the biofilms within the root canal system $[26,29,30]$. Silver nanoparticles are being evaluated for use as root canal disinfecting agents. It has been shown that $0.02 \%$ silver nanoparticle gel is able to kill and disrupt Enterococcus faecalis biofilm [31,32].

Another revolutionary introduction in the field of endodontics, the fundamental basis of which lies in nanotechnology, is bioactive glass $\left(\mathrm{SiO}_{2}-\mathrm{Na}_{2} \mathrm{O}-\mathrm{CaO}-\mathrm{P}_{2} \mathrm{O}_{5}\right.$. The use of $\mathrm{SiO}_{2}-\mathrm{Na}_{2} \mathrm{O}-\mathrm{CaO}-\mathrm{P}_{2} \mathrm{O}_{5}$ has been suggested for root canal disinfection [33]. The antimicrobial effect of bioactive glass is due its ability to maintain an alkaline environment over a period of time $[34,35]$. The efficacy of $45 \mathrm{~S} 5$ bioactive suspension - nanometric/micrometric hybrid as an antimicrobial agent showed that a ten-fold increase in silica release and 3 units of $\mathrm{pH}$ elevation was found with the nanometric bioactive glass [36].

\section{Challenges Faced by Nanodentistry}

Nanotechnology is foreseen to change health care by serving as a novel method for disease diagnosis and prevention, drug delivery and gene therapy.

Although nanotechnology appears to introduce ground breaking techniques and devices in the dental field, there are some concerns as well. These include economical nanorobot mass production technique, ethical issues and human safety, biocompatibility issues and the expertise in precise positioning and technique [26].

\section{Conclusions}

Nanotechnology will revolutionize health care, especially dentistry, more profoundly than many other developments of the past. It has the potential to bring out significant benefits, such as improved health. However, as with any other technology, it also carries a potential for misuse and abuse. The evolution of nanotechnology will help dentists with more precision made materials, drugs and equipments by which both the safety and patient compliance are enhanced.

\section{References}

1. Park B (2007) Current and Future Applications of Nanotechnology. The Royal Society of Chemistry, Cambridge, UK.

2. Feynman RP (1961) There's Plenty of Room at the Bottom. Reinhold, New York.

3. Drexler KE (1980) Engines of creation, the coming era of nanotechnology Anchor press, New York.

4. Drexler KE (1981) Molecular Engineering: An Approach to the Development of General Capabilities for Molecular Manipulation. Proc Natl Acad Sci 78: 52755278 
Citation: Sivaramakrishnan SM, Neelakantan P (2014) Nanotechnology in Dentistry - What does the Future Hold in Store? Dentistry 4: 198 doi:10.4172/2161-1122.1000198

5. Taniguchi N (1974) On the Basic Concept of Nano Technology, Proceeding of International Conference on Production Engineering. JSPE 2: 18-23.

6. Schleyer TL (2000) Nanodentistry. Fact or Fiction? J Am Dent Assoc 131: 15671568.

7. Lahn J (2006) Nano's big future: Nanotech -National geographic.

8. Kaehler T (1994) Nanotechnology: Basic concepts and definitions. Clin Chem 40: $1797-1799$

9. Rao KVP, Kumar JS (2013) Nanotechnology in dentistry. KDJ 36: 56-59.

10. Poole CP, Owens FJ (2003) Introduction to Nanotechnology. John Wiley \& Sons, New Jersey.

11. Kumar SR, Vijayalakshmi R (2006) Nanotechnology in dentistry. Indian J Dent Res 17: 62-65.

12. Rodgers $P$ (2006) Nanoelectronics. Nature Nanotech.

13. Freitas RA Jr (1999) Nanomedicine. Basic capabilities. Landes bioscience, George town, TX.

14. Freitas RA Jr (2000) Nanodentistry. J Am Dent Assoc 131: 1559-1565

15. Lampton $C$ (1995) Nanotechnology promises to revolutionize the diagnosis and treatment of disease. Genetic Engineering News 15: 23-25.

16. Whitesides GM, Love JC (2001) The art of building small. Scientific American 285: 33-41.

17. Redlich M, Katz A, Rapoport L, Wagnerb HD, Feldmanb Y, et al. (2008) Improved orthodontic stainless steel wires coated with inorganic fullerene-like nanoparticles of WS2 impregnated in electroless nickel-phosphorous film. Dent Mater 24: 1640-1646.

18. Simon Z, Watson PA (2002) Biomimetic Dental Implants - New Ways to Enhance Osseo integration. J Can Dent Assoc 68: 286-288.

19. Freitas RA Jr (1998) Exploratory design in medical nanotechnology: A mechanical artificial red cell. Artificial Cells Blood Substitute Immobile Biotechnology 26: 30-32.

20. Mjul IA, Nordahl I (1996) The density and branching of dentinal tubules in human teeth. Arch Oral Biol 41: 401-412.

21. Sumikawa DA, Marshall GN, Gee L, Marshall SJ (1999) Microstructure of primary tooth dentin. Paediatr Dent 21: 439-444.

22. Pinon-Segundo E, Ganem-Quintanar A, Alonso-Perez V, Quintanar-Guerrero D (2005) Preparation and characterization of triclosan nanoparticles for periodontal treatment. Int J Pharm 294: 217-232.
23. Freitas RA (2005) Nanotechnology, Nanomedicine and Nanosurgery. Int J Surg 3: 243-245.

24. Xu HH, Sun L, Weir MD, Takagi S, Chow LC, et al. (2007) Effects of incorporating nanosized calcium phosphate particles on properties of whisker-reinforced dental composites. J Biomed Mater Res B Appl Biomater 81: 116-125.

25. Pratap R (2005) Engaging Private Enterprise in nanotechnology research in India. ICS, Trieste.

26. Kishen A, Shi Z, Shrestha A, Neoh KG (2008) An investigation on the antibacterial and antibiofilm efficacy of cationic nanoparticulates for root canal disinfection. J Endod 34: 1515-1520.

27. Rabea El, Badawy ME, Stevens CV, Smagghe G, Steurbaut W (2003) Chitosan as antimicrobial agent: applications and mode of action. Biomacromolecules 4 : 1457-1465.

28. Rai MK, Deshmukh SD, Ingle AP, Gade AK (2012) Silver nanoparticles: the powerful nanoweapon against multidrug-resistant bacteria. J Appl Microbiol 112: 841-852.

29. Sawai J (2003) Quantitative evaluation of antibacterial activities of metallic oxide powders $(\mathrm{ZnO}, \mathrm{MgO}$ and $\mathrm{CaO})$ by conductimetric assay. J Microbiol Methods 54: 177-182.

30. Shrestha A, Shi Z, Neoh KG, Kishen A (2010) Nanoparticulates for antibiofilm treatment and effect of aging on its antibacterial activity. J Endod 36: 10301035.

31. Wu D, Fan W, Kishen A, Gutmann JL, Fan B (2013) Evaluation of the Antibacterial Efficacy of Silver Nanoparticles against Enterococcus faecalis Biofilm. J Endod.

32. Morones JR, Elechiguerra JL, Camacho A, Holt K, Kouri JB, et al. (2005) The bactericidal effect of silver nanoparticles. Nanotechnology 16: 2346-2353.

33. Zehnder M, Waltimo T, Sener B, Söderling E (2006) Dentin enhances the effectiveness of bioactive glass S53P4 against a strain of Enterococcus faecalis. Oral Surg Oral Med Oral Pathol Oral Radiol Endod 101: 530-535.

34. Sepulveda P, Jones JR, Hench LL (2002) In vitro dissolution of melt-derived $45 \mathrm{~S} 5$ and sol-gel derived 58S bioactive glasses. J Biomed Mater Res 61: 301 311.

35. Waltimo T, Brunner TJ, Vollenweider M, Stark WJ, Zehnder M (2007) Antimicrobial effect of nanometric bioactive glass 45S5. J Dent Res 86: 754757.

36. Waltimo T, Mohn D, Paqué F, Brunner TJ, Stark WJ, et al. (2009) Fine-tuning of bioactive glass for root canal disinfection. J Dent Res 88: 235-238. 